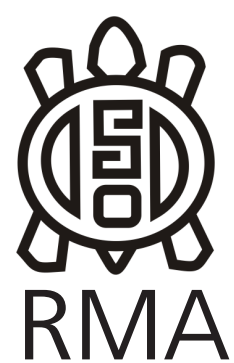

Dossier

\title{
Implementación de técnicas espectroquímicas en la investigación zooarqueológica. Cuantificación de Estroncio utilizando Laser Induced Breakdown Spectroscopy (LIBS)
}

\author{
Gabriela Srur *; Andrés D. Izeta**; Tobías Schmidt de León**; Iván \\ Cabanillas-Vidosa**; Juan C. Ferrero***; Gustavo A. Pino*** \\ *CONICET-Museo de Antropología, Facultad de Filosofía y Humanidades, \\ Universidad Nacional de Córdoba, Argentina. E-mail: Gabriela_srur@live.com.ar. \\ ${ }^{* *}$ CONICET-Museo de Antropología, Facultad de Filosofía y Humanidades, \\ Universidad Nacional de Córdoba, Argentina. E-mail:andresizeta@gmail.com. \\ ***Centro Láser de Ciencias Moleculares, INFIQC, Facultad de Ciencias Químicas, \\ Universidad Nacional de Córdoba, Argentina. E-mail: gpino@fcq.unc.edu.ar
}

\begin{abstract}
Resumen
Los estudios químicos sobre restos zooarqueológicos se vienen desarrollando con mayor intensidad en las últimas décadas, especialmente aquellos dedicados a la identificación de huellas químicas o diversos tipos de isótopos y sus relaciones. El objetivo de este trabajo es el de presentar dos procedimientos utilizados para la caracterización química de huesos en base a datos espectrométricos obtenidos mediante la técnica LIBS (Laser Induced Breakdown Spectroscopy). El primero denominado Adición Estándar, consiste en un procedimiento destructivo, con un grado de error estimable, mientras que el segundo, Reemplazo de la Matriz Ósea, constituye un método no invasivo y con un grado de error relativamente bajo. Con esto se espera lograr el desarrollo de un corpus metodológico y analítico que permita caracterizar de un modo eficiente y económico las huellas químicas de diverso material arqueofaunístico. En este sentido se intenta lograr una caracterización química de huesos arqueológicos con el fin de dar respuesta a cuestiones relacionadas tanto a la alimentación como a la movilidad de los animales en el pasado.
\end{abstract}

Palabras claves: Técnicas espectro químicas, Reemplazo de matriz ósea, Adición estándar; Laser Induced Breakdown Spectroscopy (LIBS).

Implementation of spectrochemical techniques in zooarchaeological research. Strontium quantification using Laser Induced Breakdown Spectroscopy (LIBS)

\begin{abstract}
Chemical studies on zooarchaeological remains have been most extensively developed in recent decades, especially those focused on the identification of chemical fingerprints or types of isotopes and their relationships. This paper aims at showing two procedures used for chemical characterization of bones on the basis of spectrometric data obtained by LIBS technique (Laser Induced Breakdown Spectroscopy). One called Standard Addition, a destructive procedure, with a high error degree; the other called Bone Matrix Replacement, a noninvasive method with a relatively low error degree. We expect to gather a methodological and analytical corpus to characterize, efficiently and inexpensively, the chemical fingerprints of diverse archaeofaunal material. Hence, we intend to achieve chemical characterization of archaeological bones in order to address issues related to animal eating habits and mobility in the past.
\end{abstract}

Keywords: Chemical spectrum techniques, Replacement of bone matrix, Standard Addition, Laser Induced Breakdown Spectroscopy (LIBS).

El mundo tal y cual lo conocemos presenta algunos aspectos todavía desconocidos o que no se pueden observar a simple vista, pero que sin embargo determinan nuestro modo de vida. Todo lo que nosotros logramos percibir a un nivel general, está compuesto químicamente en un mundo ordenado isotópicamente (Wada et al. 1995). Los elementos y los isótopos circulan en la biósfera y procesos como el fraccionamiento y el "mixing" producen una distribución isotópica característica y regular sobre el planeta. La cantidad de elementos e isótopos involucrados en la circulación son importantes, con grandes reservas que proveen puntos de estabilidad 
y equilibrio en la circulación de especies químicas general. Todas estas reservas de la biósfera son parte de grandes reservorios geoquímicos que circulan a lo largo del planeta sobre escalas de tiempo geológico (Bowen et al. 2009).

De este modo, las plantas y los animales registran a través de diversos mecanismos de alimentación aspectos de su ambiente en sus tejidos, y por ello pueden proveer información geográfica y climática. Para la zooarqueología, la información obtenida resulta de suma importancia para comprender procesos como prácticas alimentarias, manejo de rebaños de animales domésticos, movilidad, hábitos territoriales de las especies silvestres y estrategias socioeconómicas basadas en componentes mixtos agropastoriles (e.g. Figueroa et al. 2010, Finucane 2004, Finucane et al. 2006, Izeta et al. 2009, Izeta et al. 2010). Preguntas como ¿Cuál era su modo de vida? ¿Cómo era su dieta? ¿En qué consistía su rango de movilidad? comenzaron a ser respondidas a partir de la zooarqueología química, centrada particularmente en el estudio de relaciones isotópicas y de caracterización multielemental de muestras zooarqueológicas (e.g. Burton et al. 1999, De Niro 1985; Panarello y Fernández 2002, Szostek et al. 2009). Siguiendo algunas de estas líneas, este trabajo tiene por finalidad profundizar en el uso de procedimientos físico-químicos aplicados al registro óseo y que desemboquen posteriormente en el desarrollo de un corpus metodológico y analítico que permita responder a algunas de las preguntas mencionadas más arriba. Particularmente, se dan a conocer los primeros resultados de la aplicación de la técnica espectroquímica denominada Laser Induced Breakdown Spectroscopy (LIBS) para identificar proporciones de elementos mayoritarios y minoritarios presentes en el registro óseo arqueológico. Su relevancia radica en brindar un método potencial para realizar análisis químicos en zooarqueología.

En este caso, nos centraremos en una experiencia desarrollada con el fin de estimar la abundancia del Estroncio (Sr) que ha resultado de interés para responder preguntas sobre origen geográfico y movilidad de diversos organismos (e.g. Burton et al. 2003). Por lo anterior, el objetivo del presente trabajo es el de presentar los resultados obtenidos en la cuantificación de $\mathrm{Sr}$ en huesos de camélidos sudamericanos mediante dos aproximaciones, su comparación y la evaluación de su factibilidad técnica para su uso en muestras zooarqueológicas.

En definitiva con el testeo de estos dos modos, se espera comprobar que se pueden desarrollar métodos mínimamente invasivos para el tratamiento de muestras arqueológicas y que sean relevantes para medir la concentración multielemental dado que algunos elementos presentan mayores concentraciones cuando el consumo dominante es de carne (como cinc, estaño y cobre) o vegetales (magnesio, vanadio, manganeso, bario y estroncio) (Wing y Browen 1979, Szostek et al. 2009).

\section{Espectrometría y Láser: las bases del LIBS}

El desarrollo de la espectrometría de masas tiene su origen en el Siglo XIX con el descubrimiento de los isótopos. Ya para 1898 Wien mostró que un haz de estos iones podía ser deflectado mediante el empleo de campos eléctricos y magnéticos. En 1912, Thomson introdujo en un tubo, comúnmente empleado para determinar la relación masa-carga de iones, una pequeña cantidad de gas neón y lo atacó con un haz de electrones emitidos desde un cátodo (Ortega 2006). Éstos son capaces de colisionar y extraer algunos de los electrones externos del neón (ionización por "impacto electrónico"), produciendo así cierta cantidad de iones $\mathrm{Ne}+$. Los iones formados se aceleraban dentro del tubo con un campo eléctrico y se sometían simultáneamente a un campo magnético orientado de modo perpendicular a su trayectoria (Gross 2004). Esto permitió acceder a la cuantificación de los diversos isótopos que componen un elemento químico.

Desde hace más de treinta años, las medidas isotópicas en los espectrómetros se hacen por contaje electrónico y comparando intensidades entre los picos de la muestra y una referencia de composición conocida, de forma que el software de los equipos usa la relación $\mathrm{R}$ en sus cálculos. Es por ello, que se encuentran algunos trabajos donde se emplea directamente $\mathrm{R}$ expresada en partes por millón (Ortega 2006).

Asimismo, los Isotope Ratio Mass Spectrometer o IRMS logran mayor precisión a través de las comparaciones simples o repetidas del gas procedente de la muestra con respecto a un gas de referencia con una composición química e isotópica similar. Junto a las muestras, se introducen a diario series de estándares de composición conocida, que en la mayoría de los casos son patrones o estándares internos establecidos por cada laboratorio (Gross 2004). Sin embargo, los espectrómetros en la mayoría de los casos se diferencian sobre todo en la fuente de ionización.

Por otro lado, se desarrollaron otros métodos como Spark Source Mass Spectrometer, Inductively Coupled Plasma Mass Spectrometer y "Thermal Ionization Mass Spectrometer, los cuales miden los isótopos de elementos pesados. Además de los conocidos como Secondary Ion Mass Spectrometer (el cual realiza análisis isotópico de superficies de materiales), y los Gas Source Mass Spectrometer, familia en que se encuentran los IRMS y los Accelerator Mass Spectrometer (AMS), que se emplean cada vez más en el análisis de isótopos "no convencionales" y en datación de materiales (Ortega 2006).

Esta breve revisión de los diferentes métodos, resalta que la espectrometría de masas es destructiva a causa de que el material introducido en condiciones de vacío se altera siendo imposible su preservación posterior. 


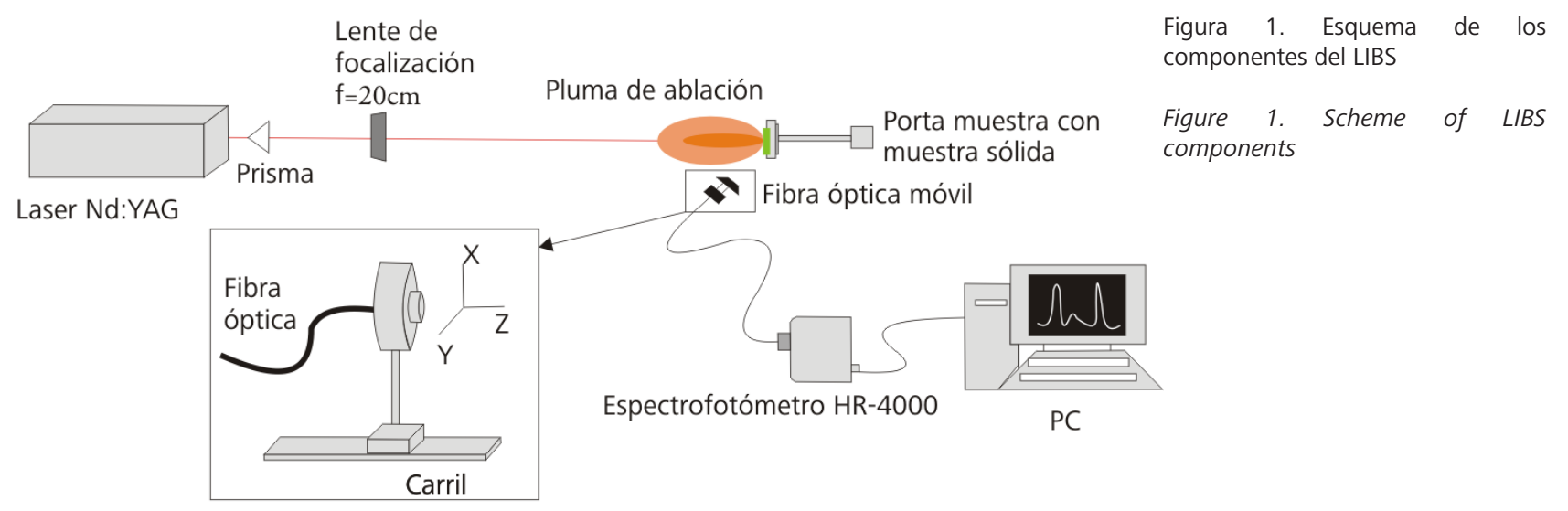

También, se han implementando otros métodos analíticos como el uso de balanzas de alta precisión o de espectroscopios de emisión óptica y espectrofotómetros de infrarrojos. Esto parece abrir la posibilidad de un futuro desarrollo de varios métodos posibles para comprender la concentración de elementos e isótopos distribuidos geo y bioquímicamente.

Por otro lado el método testeado en este trabajo tiene su precedente en el desarrollo de la técnica láser durante la década de 1960. Actualmente, el láser (Light Amplification by Stimulated Emission of Radiation) se ha convertido en una herramienta altamente versátil debido a las ventajas que ofrece como direccionalidad, monocromaticidad, potencia y coherencia temporal (Capello et al. 2009). Al ser un método sensible y selectivo, se lo emplea para detectar e identificar concentraciones de elementos químicos en diferentes muestras.

Entonces el LIBS (Figura 1) consiste principalmente en un láser pulsado de alta energía focalizado en una superficie para ablacionar el material y crear un plasma (o pluma de ablación), el cual está formado por una mezcla de especies como iones, electrones, átomos, agregados y clusters que se encuentran a muy elevada temperatura (generalmente superan los 10000 K) (Capello 2009). Debido a ello y a la resolución de los espectrómetros asociados al sistema en la determinación y cuantificación son consideradas todas las especies de cada elemento. Por otro lado y de modo complementario el LIBS permite el análisis de multielementos (Capello 2009) con lo que su utilización para identificar huellas químicas lo convierte en una técnica apropiada para diversos estudios zooarqueológicos.

\section{Materiales y métodos}

La muestra seleccionada sobre la que se planteó determinar la concentración de Sr mediante LIBS corresponde a dos elementos óseos (fémur y húmero) de Lama glama (Ilama) moderna procedentes del área denominada Casas Coloradas, Valle del Cajón, departamento de Santa María (ver Figura 2). La región se ubica en el extremo nor-oriental de la Provincia de
Catamarca (Departamento de Santa María) (Izeta 2007).

Sobre esta muestra se procedió a la implementación de dos aproximaciones con el fin de contrastar a través de los resultados cuantitativos cuál de ellas nos aportaba una mejor definición en cuanto al cálculo de la abundancia de este elemento químico dentro de esta matriz ósea.

En principio cada una de las aproximaciones consistió en la preparación de muestras con el fin de construir curvas de calibración del elemento Sr. Una utilizando como matriz el tejido compacto tanto del fémur como del húmero y otro en el que la curva se basó en la utilización de una matriz de carbonato de calcio $\left(\mathrm{CO}_{3} \mathrm{Ca}\right)$. En ambos se confeccionaron pastillas a las que se les fue agregando valores conocidos de $\mathrm{Sr}$ con el fin de poder cuantificarlo. Luego se compararon estos resultados de la curva calibrada con espectros obtenidos a partir de mediciones directas en los huesos de llama. En este último caso los huesos no tuvieron ninguna preparación previa.

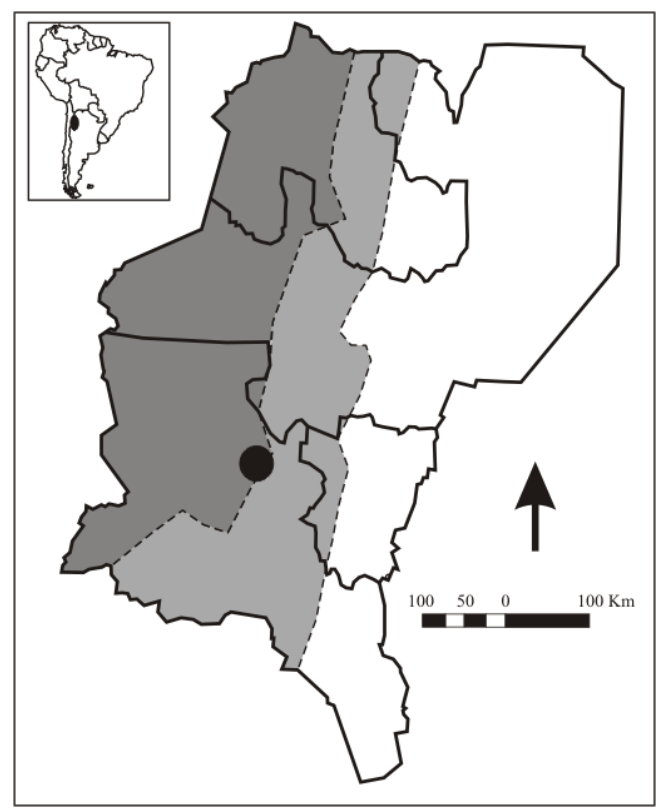

Figura 2. Procedencia de las muestras de Lama glama.

Figure 2. Provenience of the Lama glama sample used in this work 
La primera aproximación se denomina Adición de estándar y consiste en preparar una matriz de control compuestas por muestras a partir de hueso molido, adicionando a cada una concentraciones crecientes y conocidas de Sr. Cabe destacar que la aplicación de este método es destructiva dado de que es necesario que se pulverice el hueso para preparar la muestra, la cual se someterá al análisis LIBS, obteniéndose una curva de calibración de intensidad de emisión de Sr en función de la concentración. Esto genera una gráfica la cual permite visualizar la concentración de Sr en la muestra ósea comparativa.

Por otro lado es importante notar que esto debe hacerse sólo una vez para cada muestra comparativa que en general puede ser actual y no necesariamente arqueológica, ya que lo que se quiere obtener es un patrón químico georreferenciado con fines comparativos. En este caso la curva de calibración de Sr obtenida puede ser utilizada como patrón para los elementos óseos arqueológicos obtenidos de excavaciones del Valle del Cajón.

La segunda aproximación recibe el nombre de Remplazo de matriz ósea y está caracterizada por emplear una técnica no destructiva, la cual radica en la obtención de una curva de calibración de modo análogo al anterior, pero en vez de utilizar hueso (moderno o arqueológico) se utiliza como reemplazo una muestra de carbonato de calcio $\left(\mathrm{CO}_{3} \mathrm{Ca}\right)$, uno de los componentes principales en la estructura del hueso. Posteriormente, se somete al análisis LIBS la pieza original, donde el daño a su superficie es mínimo.

En cuanto a la preparación de la muestra se realizaron varios pasos. El primero consiste en la limpieza superficial, para eliminar posibles contaminantes del espécimen óseo (tierra, material orgánico extra a la estructura ósea, etc.). Esto es realizado por medios mecánicos y químicos en una superficie no mayor a los $5 \mathrm{~mm}^{2}$, la cual es separada del resto del elemento, obteniendo un fragmento cuyo peso medio es de aproximadamente 1 a 2 gramos.

El segundo, consiste en la reducción en tamaño del fragmento para su molido final, obteniendo un polvo cuyo particulado se estima en 0,3 $\mathrm{mm}$ de diámetro.

Cabe subrayar que para la segunda aproximación (Reemplazo de la matriz ósea), la muestra se preparó siguiendo los mismos pasos que para la metodología anterior, sólo que se utiliza en vez de hueso molido una muestra de $\mathrm{CO}_{3} \mathrm{Ca}$, con el fin de no utilizar la matriz ósea y de este modo evitar su destrucción. La diferencia principal, es que en este caso y adicionalmente, debe registrarse el espectro LIBS de la muestra ósea, en su estado original y sin ninguna preparación, y en las mismas condiciones experimentales (energía del láser de ablación) en que se registran los espectros LIBS de las pastillas de $\mathrm{CO}_{3} \mathrm{Ca}$ (empleadas para la construcción de la curva de calibración).

Posteriormente, se preparan diez tubos de ensayo, donde se colocan $0,5 \mathrm{~g}$ de la muestra ósea, agregando a cada uno, una concentración conocida y creciente de solución de Sr. Estas muestras son sometidas a la estufa durante 12 horas con el fin de eliminar la humedad proveniente de la solución de Sr.

Luego la muestra de cada tubo es mortereada para cumplimentar con dos objetivos: homogeneizar su contenido y reducir el particulado formado por la nueva agregación de la matriz ósea, a raíz de la adición de la solución de Sr.
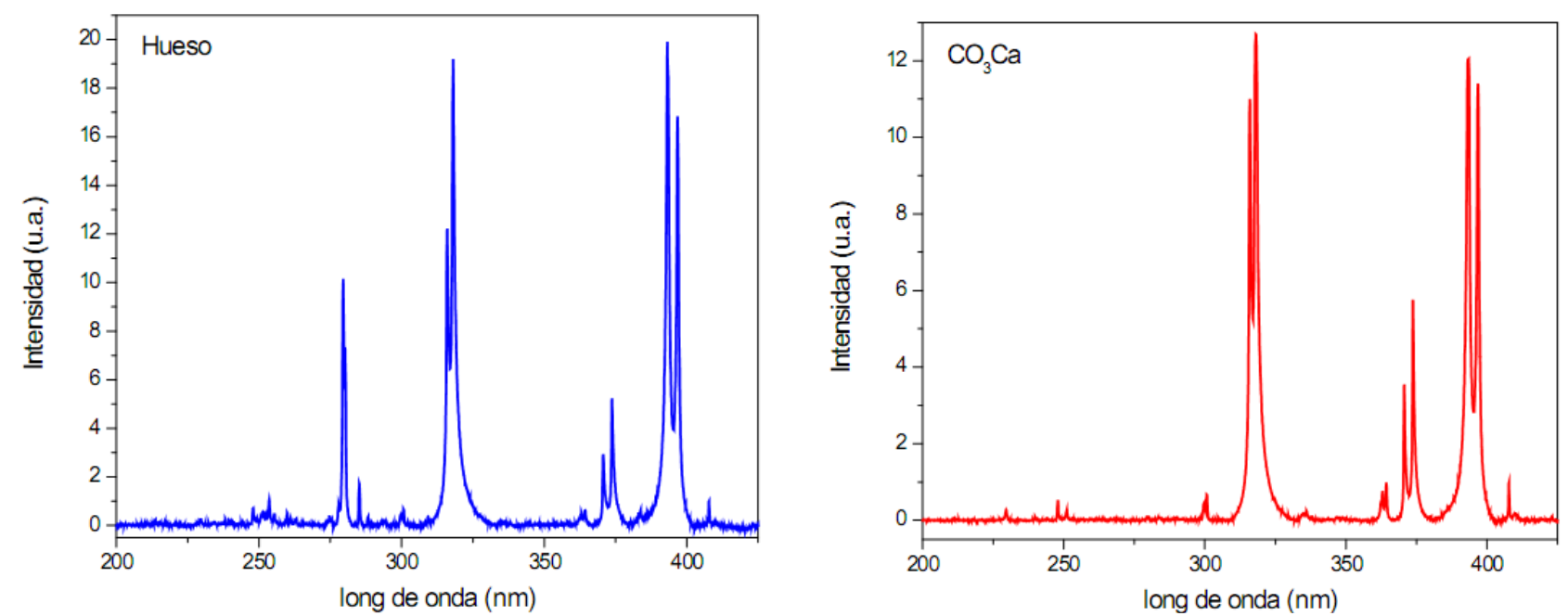

Figura 3. Espectros proveniente de la matriz ósea y del registrado a partir de $\mathrm{CO}_{3} \mathrm{Ca}$, se destacan las líneas de emisión empleadas para cuantificar (Sr) y normalizar (Ca).

Figure 3. Spectra from bone matrix and that from $\mathrm{CO}_{3} \mathrm{Ca}$; emission lines used to quantify (Sr) and normalize (Ca) are noticeable. 

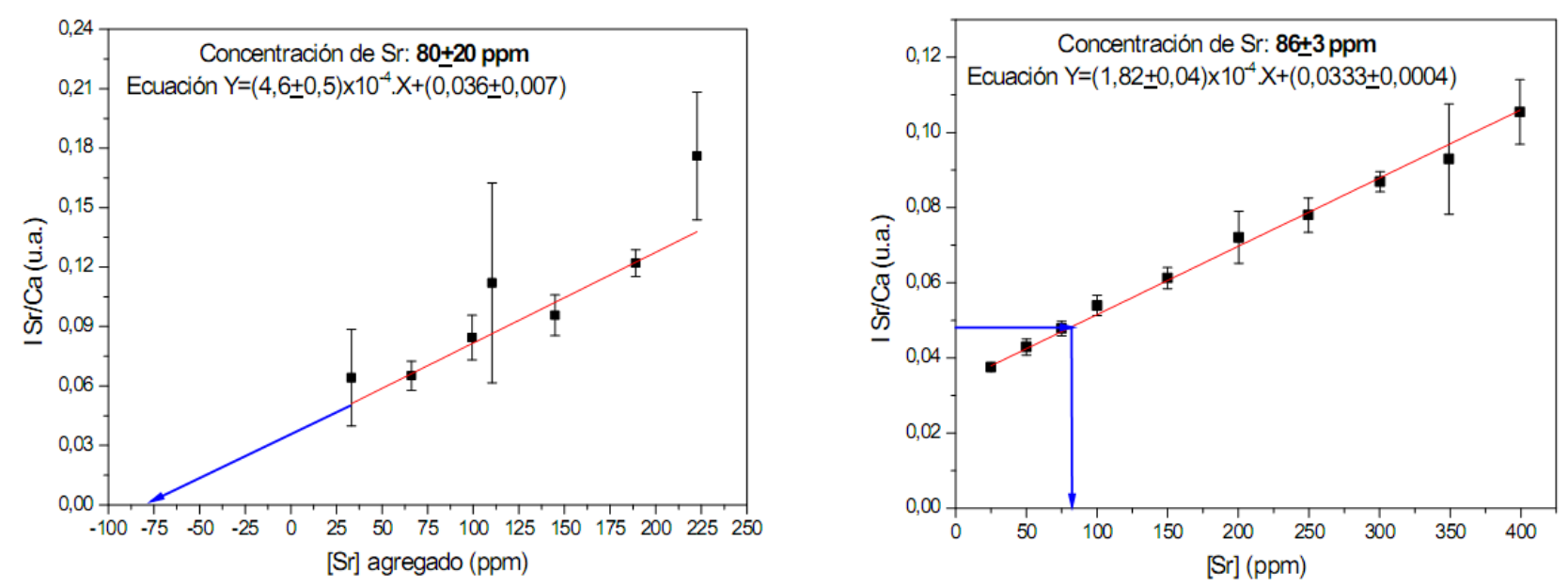

Figura 4. Resultados de las dos aproximaciones: Reemplazo de matriz ósea y adición estándard.

Figure 4. Results from two approaches: Bone matrix replacement and Standard Addition.

A continuación y a partir de cada muestra, se elaboran tres "pastillas" por compresión, sometiendo una porción de la muestra a una presión de 4 toneladas. Por último y con las 3 pastillas, se obtuvieron cinco espectros LIBS.

\section{Resultados}

Cada espectro obtenido se normalizó en intensidad, y para ello, se empleó la emisión correspondiente a un elemento contenido en alta proporción en la matriz (sea en hueso o $\mathrm{CO}_{3} \mathrm{Ca}$ ), como es el Calcio (Ca).

Si bien existe una gran similitud entre el espectro proveniente de la matriz ósea y del registrado a partir de $\mathrm{CO}_{3} \mathrm{Ca}$, se destacan en cada aproximación las líneas de emisión empleadas para cuantificar (Sr) y normalizar (Ca) como se observa en la Figura 3.

De este modo, con el método de Adición Estándar, se determinó que la concentración de $\mathrm{Sr}$ en hueso es de $80 \pm 20$ ppm. Si bien los resultados son de interés, se observó que la metodología posee un 30\% de error en la determinación. Aún así, el principal objetivo de este análisis, fue tener una referencia para cotejar el valor obtenido por la metodología donde no se utiliza la matriz ósea y si se utiliza una matriz compuesta por $\mathrm{CO}_{3} \mathrm{Ca}$.

En el caso del método de Reemplazo de matriz ósea, la concentración de Sr obtenida es de $86 \pm 3$ ppm. El valor coincide con el obtenido a través del análisis destructivo de Adición Estándar, pero en este caso, el error asociado a la determinación es sólo de un 3,5\% (Figura 4).

De este modo, se observa que el método de Reemplazo de matriz ósea, cumple con dos objetivos simultáneamente: por un lado la preparación de la matriz de control no utiliza hueso (moderno o arqueológico) y por lo tanto no destruye la muestra, por otro el daño que produce a la muestra arqueológica que debe ser medida es ínfimo dado de que se cuenta con un equipo de rotación de la muestra para que en cada pulso la superficie sea nueva, y en consecuencia sólo produce pequeños cráteres en la superficie tratada que son imperceptibles al ojo humano.

\section{Conclusiones}

Por lo presentado a lo largo de este trabajo la aplicación de estudios físico químicos sobre muestras arqueológicas nos permiten indagar en diversas cuestiones relacionadas entre otras temáticas con la alimentación y la movilidad. En este sentido poder acercarnos a la cuantificación de elementos presentes en especímenes óseos permite comenzar a caracterizarlos a partir de distintas especies de elementos químicos y con ello lograr una identificación más ajustada de los posibles procesos por los cuales se generaron los huesos. Con ello se espera poder dar respuesta a los interrogantes planteados anteriormente utilizando una aproximación que incluye instrumentos analíticos que no demandan una inversión importante de material arqueológico a ser procesado y que puede realizar mediciones sin preparaciones previas muy elaboradas como si lo exigen otras metodologías. De este modo, la aproximación que denominamos reemplazo de la matriz ósea por $\mathrm{CO}_{3} \mathrm{Ca}$ demuestra ser apropiado para la determinación de Sr en huesos de camélidos. Por supuesto esta cuantificación debe asociarse con la de otros elementos, pero la técnica demostró ser apropiada para alcanzar este objetivo. En este sentido se prevé una agenda futura en la cual es necesario efectuar curvas de calibración para otros elementos diagnósticos como Bario (Ba) y Plomo (Pb), los cuales son importantes en zooarqueología porque permiten en su conjunto reconstruir paleodietas y reconocer la variabilidad geográfica donde los animales interactuaban junto con los humanos.

Córdoba, 7 de Agosto de 2012 


\section{Bibliografía}

Bowen, G.J.; West, J.B.; Vaughn, B.H.; Dawson, T.E.; Ehleringer, J.R.; Fogel, M.L.; Hobson, K.A.; Hoogewerff, J.; Kendall, C.; Lai, C.T.; Miller, C.C.; Noone, D.; Schwarcz, H.P. y Still, C.J. 2009. Isoscapes to address large-scale Earth science challenges. Eos 90: 109-116.

Burton, J.H.; T.Price y W. D. Middelton. 1999. Correlation of bone $\mathrm{Ba} / \mathrm{Ca}$ and $\mathrm{Sr} / \mathrm{Ca}$ due to biological purification of calcium. Journal of Archaeological Science 26:609-16 Burton, J., T. Price, L. Cahuee y L. Wright. 2003. The use of barium and strontium abundances in human skeletal tissues to determine their geographic origins. International Journal of Osteoarchaeology 13:88-95.

Capello, C. 2009. Desarrollo y puesta a punto de técnicas espectroquímicas: Cuantificación de Sr en hueso mediante LIBS. Practicanato profesional de la Licenciatura en Química. Facultad de Ciencias Químicas, UNC.

Capello M. C.; I. Cabanillas-Vidosa; A. Izeta; J. C. Ferrero y G. A. Pino. 2009. Cuantificación de Sr en muestras óseas arqueológicas por medio de Ablación Láser Pulsada acoplada a Espectroscopia de Emisión Óptica (LAP-OES). Trabajo presentado en el XVI Congreso Argentino de Fisicoquímica y Química Inorgánica. Salta, 18 al 21 de mayo de 2009.

De Niro, M.J. 1985. Postmortem preservation and alteration of in vivo bone collagen isotope ratios in relation to paleodietary reconstruction. Nature 317: 806-809.

Figueroa, G. G.; M.Dantas y A. Laguens. 2010. Practicas agropastoriles e innovaciones en la producción de plantas y animales en los Andes del Sur. El Valle de Ambato, Argentina, primer milenio d.C. International Journal of South American Archaeology 7: 6-13.

Finucane, B. 2004. Isotopes and animal management at Conchopata, Perú. Unpublished Master Dissertation. School of Archaeology, Oxford University. URL:WwW. rlaha.ox.ac.uk/people/BF2-1.pdf

Finucane, B., P. Maita Agurto y W. H. Isbell. 2006. Human and animal diet at Conchopata, Peru: stable isotope evidence for maize agriculture and animal management practices during the Middle Horizon, Journal of Archaeological Science 33: 1766-1776

Gross, J.H. 2004. Mass Spectrometry. A textbook. Springer. $518 \mathrm{pp}$

Izeta, A. D. 2007. Zooarqueología del sur de los valles Calchaquies (Provincias de Catamarca y Tucumán, República Argentina): Análisis de conjuntos faunísticos del primer milenio A.D. B.A.R. International Series S1612. John and Erica Hedges, Oxford, Inglaterra

Izeta, A. D., A. G. Laguens, B. Marconetto y M. C. Scattolin. 2009. Camelid use in Meridional Andes during first millennium A.D. A preliminary approach using stable isotopes. International Journal of Osteoarchaeology 19: 204-214.

Izeta, A. D., M. Dantas, M. G. Srur, M. B.Marconetto y A. G. Laguens. 2010. Isótopos estables y manejo alimentario de camélidos durante el primer milenio A.D. en el valle de Ambato (noroeste argentino). En Bertolino, S, Cattaneo G. R y A. D. Izeta (Eds) La arqueometría en Argentina y Latinoamérica. Editorial de la FFyH, Córdoba.

Ortega R. R. 2006. Fundamentos teóricos y técnicos de los isótopos estables. En: http://www.ruena.csic.es/pdf/ ponencia_ruena_2006_redondo.pdf.

Panarello, O.H. y J. Fernández. 2002. Stable isotope carbon isotope measurements on hair from wild animals from altiplanic environments of Jujuy. Radiocarbon 44: 709-716.

Szostek, K.; H. Glab y A. Pudlo. 2009. The use of strontium and barium analyses for the reconstruction of the diet of the early medieval coastal population of Gdansk (Poland): A preliminary study. HOMO-Journal of Comparative Human Biology 60: 359-372.

Wada, E., T. Yoneyama, M. Minagawa, T. Ando, and B.D. Fry (eds.), 1995. Stable Isotopes in the Biosphere. Kyoto University Press, Japan.

Wing, E. S. y A. B. Brown. 1979. Paleonutrition. Method and theory in prehistoric foodways. Academic Press. New York. 